\title{
Do CTRC mutations affect the development of alcoholic chronic pancreatitis and its course among Poles: Preliminary study
}

\author{
Halina Cichoż-Lach ${ }^{1, A-D}$, Małgorzata Michalak-Wojnowska ${ }^{2, A-C}$, Emilia Lis-Janczarek ${ }^{1, B-D}$, \\ Jacek Wojcierowski ${ }^{3, E, F}$, Marcin Hydzik $3, B, C$ \\ ${ }^{1}$ Department of Gastroenterology, Medical University of Lublin, Poland \\ 2 Department of Cancer Genetics, Medical University of Lublin, Poland \\ ${ }^{3}$ Genetic Testing Laboratory, Lublin, Poland \\ A - research concept and design; $\mathrm{B}$ - collection and/or assembly of data; $\mathrm{C}$ - data analysis and interpretation; \\ $D$ - writing the article; $E$ - critical revision of the article; $F$ - final approval of the article
}

Address for correspondence

Halina Cichoż-Lach

E-mail: lach.halina@wp.pl

Funding sources

None declared

Conflict of interest

None declared

Received on March 19, 2017

Reviewed on May 2, 2017

Accepted on July 28, 2017

Published online on 0ctober 2, 2018

Cite as

Cichoż-Lach H, Michalak-Wojnowska M, Lis-Janczarek E, et al. Do CTRC mutations affect the development of alcoholic chronic pancreatitis and its course among Poles: Preliminary study. Adv Clin Exp Med. 2019;28(3):307-312. doi:10.17219/acem/76130

DOI

10.17219/acem/76130

\section{Copyright}

Copyright by Author(s)

This is an article distributed under the terms of the

Creative Commons Attribution Non-Commercial License

(http://creativecommons.org/licenses/by-nc-nd/4.0/)

\begin{abstract}
Background. Genetic mutations are one of the etiological factors that predispose people to develop chronic pancreatitis.

Objectives. The aim of our study was to examine the effect of p.Trp55*, p.Arg254Trp and c.738_761del mutations in the chemotrypsin gene (CTRC) on the development of alcoholic chronic pancreatitis (ACP) in order to answer the questions whether these mutations vary between gender groups, whether they were related to the age when ACP was first diagnosed, and whether they affected the morphological changes in the pancreas and the course of ACP.
\end{abstract}

Material and methods. The study included 124 patients with ACP, 52 with nonalcoholic pancreatitis and 52 controls. The p.Trp55*, c.738_761del and p.Arg254Trp mutations in the CTRC gene were tested by the polymerase chain reaction (PCR).

Results. The c.738_761del and p.Arg254Trp mutations occurred in 3.07\% and 1.31\% of cases, respectively. None of the examined patients were found to have the p. Trp $55^{*}$ mutation. The frequency of detected mutations did not significantly differ between the study groups. The c.738_761del mutation was detected more frequently in women than in men. No significant differences were found in the age at ACP onset, morphological changes affecting the pancreas, or in the course of ACP between the patients with and without the 2 examined mutations. The c.738_761del mutation was significantly more frequent in the diabetic patients than in the non-diabetics. The patients with this mutation more frequently required surgery than those without the c.738_761del mutation.

Conclusions. No relationship between the c.738_761del and p.Arg254Trp mutations and the development of APC was found. The c.738_761del mutation was more frequent in females than in males. Neither mutation affected the patient's age at ACP onset or its course. In contrast to p.Arg254Trp, the c.738_761del mutation correlated with diabetes development and the need for surgery in the course of ACP.

Key words: alcohol, alcoholic chronic pancreatitis, CTRC mutations 


\section{Introduction}

Chronic pancreatitis $(\mathrm{CP})$ can present as recurrent episodes of acute inflammation or as progressive inflammatory conditions that result in fibrosis, calcification or altered morphology of the pancreas, the consequence of which is endocrine and exocrine failure of the organ. ${ }^{1,2}$ Global annual incidence rates of CP range from 5 to 12 cases per 100,000 people; the prevalence of $\mathrm{CP}$ is about 50 cases per 100,000 people. ${ }^{3}$ The disease occurs with a varied geographic distribution. In developed and western European countries, CP is generally due to alcohol consumption (38\% of men and $11 \%$ of women). In the USA, Italy and Denmark, more than $50 \%$ of cases are alcohol-related, but in Brazil, the proportion reaches $89.6 \%{ }^{4,5}$ Alcohol consumption has been increasing in developing countries (China and India) due to rapid urbanization and increased affluence; therefore, this rise is expected to increase the burden of alcoholrelated pancreatitis in these countries. ${ }^{6}$

Among many etiological factors, the role of genetic predisposition to $\mathrm{CP}$ is clearly highlighted. The genetic factors of CP include a mutation/polymorphism of the cationic trypsinogen gene (PRSS1), the serine protease inhibitor Kazal type 1 gene (SPINK1), the chemotrypsin gene (CTRC), the cystic fibrosis transmembrane conductance regulator gene $(C F T R)$, and the calcium sensing receptor gene (CASR). CASR is responsible for calcium homeostasis and some patients with hypercalcemia develop CP. This observation may encourage one to postulate that a mutation in the CASR gene may favor CP. Polymorphisms of interleukin-encoding genes, such as tumor necrosis factor-alpha (TNF- $\alpha$ ), transforming growth factor-beta-1 (TGF- $\beta 1$ ), interferon-gamma (INF- $\gamma$ ), vascular endothelial growth factor (VEGF), and intercellular adhesion molecule 1 (ICAM-1), are also major genetic contributors to the development of CP. ${ }^{7,8}$ In 2013, the carboxypeptidase A1 (CPA1) gene was identified as a novel gene of pancreatitis susceptibility. ${ }^{7,9,10}$ According to studies carried out on Polish individuals, the NS34 mutation of the SPINK1 gene seems to be significantly correlated with alcoholic chronic pancreatitis (ACP). ${ }^{11}$

The CTRC gene encodes chemotrypsin C, a digestive enzyme produced by lobular pancreatic cells. Prematurely activated trypsin is destroyed by $C T R C$, which acts on the molecule within the calcium-binding loop in the absence of calcium, and therefore is a crucial candidate gene in the pathogenesis of CP. Since trypsin degradation serves as a protective mechanism against pancreatitis, it may be hypothesized that a loss of function in trypsin-degrading enzymes increases the risk for pancreatitis. ${ }^{12,13}$ Numerous CTRC mutations and polymorphisms have been presented so far. However, the p.Trp55\%, p.Arg254Trp and c.738_761del mutations at exon 7 have not been studied among Poles with ACP, which poses a serious clinical challenge.

The aim of this study was to examine the effect that the p.Trp55*(W55X), p.Arg254Trp (R254W) and c.738_761del (del24) mutations in the CTRC gene have on the development of ACP, and to answer the question whether these mutations vary between gender groups. Moreover, we wanted to learn whether the mutations were related to the age when ACP was first diagnosed, and whether they affected the morphological changes in the pancreas and the course of ACP. The focus was on the 3 above-mentioned mutations of $C T R C$, because it seems that they have the greatest impact on the development of $\mathrm{CP}$ and are the most frequently observed mutations in exon $7 .^{7}$

\section{Material and methods}

\section{Material}

The study group was comprised of a homogenous Caucasian ethnic group of 228 patients, including 124 with ACP, 52 with nonalcoholic $\mathrm{CP}(\mathrm{NCP})$, and 52 healthy volunteers who made up the control group.

Chronic pancreatitis diagnosis was based on the standard criteria: a clinically confirmed history of recurrent episodes of acute pancreatitis and the results of imaging tests on the pancreatic structure (ultrasonography - USG, computed tomography - CT, endoscopy, or endoscopic retrograde cholangiopancreatography), such as calcifications, fibrosis of the pancreatic parenchyma, intraductal calcifications, and widened or irregular pancreatic ducts. Histological tests confirmed the diagnosis in 3 patients with ACP; in other cases, a biopsy was not performed. Alcoholic etiology was established on the basis of a medical history, i.e., the consumption of $80 \mathrm{~g}$ of pure ethanol in a 24-h period (males) or $>40 \mathrm{~g}$ of pure ethanol in a $24-\mathrm{h}$ period (females) in the previous 2 years or more. In addition, diabetes was diagnosed in 53 patients with ACP and insulin-dependent diabetes was diagnosed in 27 patients. Patients with a biliary, toxic, metabolic, or family history of CP were excluded from this group.

The group of patients with NCP included idiopathic CP cases. Chronic pancreatitis was diagnosed on the basis of the criteria presented above. The patients with an alcoholic, toxic, metabolic, or family history of CP were excluded from the study group. Four patients suffered from type 2 diabetes, treated with oral medication.

The control group was comprised of healthy volunteers with no history of alcohol consumption. They did not present any clinical symptoms or abnormalities on abdominal USG. There were no episodes of acute pancreatitis in this study group. Table 1 presents the characteristics of the study groups.

To examine whether there is an association between the p.Trp55\%, p.Arg254Trp and c.738_761del mutations in the CTRC gene and the development of ACP, we compared the frequencies of the p.Trp55\%, p.Arg254Trp and c.738_761del mutations in the CTRC gene in our 3 groups of patients: with ACP, with NCP, and in healthy controls. 
Table 1. Characteristics of the study groups

\begin{tabular}{|l|c|c|c|c|c|c|c|}
\multirow{2}{*}{ Patient group } & $\begin{array}{c}\text { Number } \\
\text { of patients }\end{array}$ & \multicolumn{2}{|c|}{ Gender } & Age [years] & $\begin{array}{c}\text { Presence } \\
\text { of diabetes [n] }\end{array}$ & $\begin{array}{c}\text { Mean BMI } \\
{\left[\mathrm{kg} / \mathrm{m}^{2}\right] \pm \text { SD }}\end{array}$ \\
\cline { 3 - 8 } & 124 & 34 & 90 & mean \pm SD & $43.07 \pm 9.04$ & 53 & $24.32 \pm 4.35$ \\
ACP & 52 & 28 & 24 & $39.97 \pm 11.03$ & 4 & $25.45 \pm 5.97$ \\
NCP & 52 & 24 & 28 & $40.38 \pm 7.88$ & 0 & $24.67 \pm 3.47$ \\
\hline Controls & 52 & & \\
\hline
\end{tabular}

CP - chronic pancreatitis; ACP - alcoholic chronic pancreatits; NCP - nonalcoholic chronic pancreatitis; F - female, M - male; BMI - body mass index; SD -standard deviation.

The study protocol was approved by the local Ethical Committee (No. 131/2013) (Medical University of Lublin, Poland), and all participants gave written informed consent to participate in the study.

\section{Methods}

The p.Trp55\%, c.738_761del and p.Arg254Trp mutations in the CTRC gene were tested in all patients.

\section{DNA isolation}

DNA was isolated from peripheral blood leukocytes, using a Blood DNA Purification Kit (EURx, Gdańsk, Poland) according to the instructions of the manufacturer. The lymphocytes were separated by the Ficoll gradient technique.

\section{Determination of the p.Trp55* mutation in the CTRC gene}

Polymerase chain reactions (PCRs) were performed with $100 \mathrm{ng}$ of genomic DNA in a total volume of $20 \mu \mathrm{L}$, using a Biometra T Personal thermal cycler (Biometra, Gottingen, Germany). DNA was amplified with the primers named p.Trp $55^{*}$ F F and p.Trp $55^{*}$ R (Table 2), designed by the Primer3 application in the Genetic Testing Laboratory in Lublin, Poland, and with a Taq PCR Master Mix kit (EURx), according to the manufacturers' instructions. The setting parameters are listed in Table 2.

The PCR products were digested overnight at $37^{\circ} \mathrm{C}$ in a CLN 15 STD INOX/G incubator by the restriction enzyme PmlI (New England Biolabs, Ipswich, USA).
The composition of the $20.4 \mu \mathrm{L}$ restriction mix was $12 \mu \mathrm{L}$ of the PCR product, $5 \mathrm{U}$ of PmlI, $2 \mu \mathrm{L}$ of CutSmart buffer, and $6 \mu \mathrm{L}$ of water. Fifteen microliters of the digestion reaction products were used for electrophoresis. The products of digestion were separated in 3\% agarose gel (Sigma Aldrich, St. Louis, USA), stained with Simply Safe (EURx), and visualized on a transilluminator (JW Electronic, Warszawa, Poland). The wild alleles were digested into fragments of $202 \mathrm{bp}$ and $262 \mathrm{bp}$; the mutated allele did not have a restriction site and was identified as a band of $462 \mathrm{bp}$. To confirm the results, the analyzed samples underwent sequencing in both directions by means of primers 3R and 3F (Table 2), using a BigDye Terminator 3.1 kit (Life Technologies, Carlsbad, USA). The products of sequencing were electrophoretically separated on an ABI3100 sequencer, on a 50-centimeter capillary in the POP6 polymer, and analyzed by the Sequencing Analysis (Applied Biosystems, Foster City, USA).

\section{Determination of the c.738_761del and p.Arg254Trp mutations in the CTRC gene}

Both mutations were determined in 1 assay by using the same primer pairs, named c.738_761del_F and c.738_761del_R (Table 2), designed by Primer3 in the Genetic Testing Laboratory in Lublin, Poland. The PCR amplification was performed using a Biometra T Personal thermal cycler (Biometra) in a volume of $20 \mu \mathrm{L}$ with $100 \mathrm{ng}$ of genomic DNA, 2 primers and a Taq PCR Master Mix kit (EURx), according to the manufacturers' instructions. The setting parameters are listed in Table 2. Following that, the PCR products were digested overnight at $37^{\circ} \mathrm{C}$

Table 2. Primers used to determine the p.Trp55*, p.Arg254Trp and c.738_761del mutations in the CTRC gene

\begin{tabular}{|c|c|c|c|c|}
\hline Application & Primer name & Primer sequence & $\begin{array}{l}\text { Annealing } \\
\text { temperature }\end{array}$ & $\begin{array}{l}\text { Product } \\
\text { length }\end{array}$ \\
\hline \multirow{4}{*}{ PCR-RFLP } & p.Trp55*_F & 5' AGCCCTATTCACTGGTTCTTCTG 3' & \multirow{2}{*}{$59^{\circ} \mathrm{C}$} & 464 bp \\
\hline & p.Trp55*_R & 5'CAACTGAGTTACTGGGTGTGAGTAG 3' & & \\
\hline & c.738_761del_F & 5' TGGTGGCTTATGCCCTCCCG 3' & \multirow{2}{*}{$59^{\circ} \mathrm{C}$} & $209 \mathrm{bp}$ \\
\hline & c.738_761del_R & 5' GGACAGCTGTGGAGGCAGCAC 3' & & \\
\hline \multirow{4}{*}{ Sequencing } & CTRC_3F & $5^{\prime}$ ACCTGCAGGCTGACACACA $3^{\prime}$ & \multirow{2}{*}{$59^{\circ} \mathrm{C}$} & $325 \mathrm{bp}$ \\
\hline & CTRC_3R & 5' GCTGGTTCCTGGCACATAAT 3' & & \\
\hline & CTRC_7F & 5' GCAGGCTGAGGCCAAAT 3' & \multirow{2}{*}{$58^{\circ} \mathrm{C}$} & $467 \mathrm{bp}$ \\
\hline & CTRC_7R & 5' TGAATGAGTGACTGAATAAGTG 3' & & \\
\hline
\end{tabular}

PCR-RFLP - polymerase chain reaction-restriction fragments length polymorphism; both mutations, c.738_761del and p.Arg254Trp, were determined by using the same primers (C.738_761del_F and C.738_761del_R). 
in a CLN 15 STD INOX/G incubator by the restriction enzyme SmaI (New England Biolabs). The composition of the $20.36 \mu \mathrm{L}$ restriction mix was $12 \mu \mathrm{L}$ of the PCR product, $10 \mathrm{U}$ of SmaI, $1.6 \mu \mathrm{L}$ of CutSmart buffer, $0.16 \mu \mathrm{L}$ of bovine serum albumin (BSA), and $6 \mu \mathrm{L}$ of water. Fifteen microliters of the digestion reaction products were used for electrophoresis. The products of digestion were separated in 3\% agarose gel (Sigma Aldrich), stained with Simply Safe (EURx) and visualized on a transilluminator (JW Electronic). The wild allele was digested into fragments of 152 bp and $57 \mathrm{bp}$; the mutated allele (p.Arg254Trp) did not have restriction sites and appeared as a band of $209 \mathrm{bp}$. In the case of the c.738_761del mutation, the wild allele was seen as a band of $209 \mathrm{bp}$, but a product of $185 \mathrm{bp}$ in length corresponded to the allele with a 24-bp deletion. To confirm the results, the analyzed samples underwent sequencing in both directions by means of primers $7 \mathrm{~F}$ and $7 \mathrm{R}$ (Table 2) (the same primers for both mutations), using a BigDye Terminator 3.1 kit (Life Technologies) according to the manufacturer's instructions. The products of sequencing were electrophoretically separated on an ABI3100 sequencer, on a 50-centimeter capillary in the POP6 polymer, and analyzed by the Sequencing Analysis (Applied Biosystems) according to the manufacturer's instructions.

\section{Statistical analysis}

To describe the quantitative characteristics, the average values with standard deviations (SDs) were used. The comparison of the age at ACP onset was analyzed by Student's t-test for independent samples. The qualitative data was described as numbers. To compare the frequency of the
p.Trp55*, c.738_761del and p.Arg254Trp mutations in the CRTC gene between the study groups, the ANOVA test was performed. To compare the frequency of the examined mutations in the CRTC gene between women and men, the $\chi^{2}$ test with Yates's correction or Fisher's exact test was used, as appropriate. Statistical significance between the differences was assumed at $\mathrm{p}<0.05$. All calculations were done by means of STATISTICA PL software (StatSoft, Kraków, Poland).

\section{Results}

The frequency of the examined mutations in the study groups is presented in Table 3 . In the group of 228 patients, the c.738_761del mutation occurred in $3.07 \%$ of cases and the p.Arg254Trp mutation was found in $1.31 \%$ of patients (Fig. 1,2). In none of the examined patients was the p.Trp $55^{*}$ mutation detected. All mutations were heterozygotic. The c.738_761del mutation was detected only in the group of patients with ACP (4.35\%); the p.Arg254Trp mutation was found in $1.45 \%$ of patients with ACP and in $1.92 \%$

Table 3. The frequency of the examined mutations in the CRTC gene in the study groups

\begin{tabular}{|l|c|c|c|c|}
\hline \multicolumn{1}{|c|}{ Mutation } & ACP & NCP & Controls & p-value \\
\hline p.Trp55* & $0 / 124$ & $0 / 52$ & $0 / 52$ & NS \\
\hline c.738_761del & $7 / 124$ & $0 / 52$ & $0 / 52$ & NS \\
\hline p.Arg254Trp & $2 / 124$ & $0 / 52$ & $1 / 52$ & NS \\
\hline
\end{tabular}

ACP - alcoholic chronic pancreatitis; NCP - nonalcoholic chronic pancreatitis; NS - no statistically significant differences.

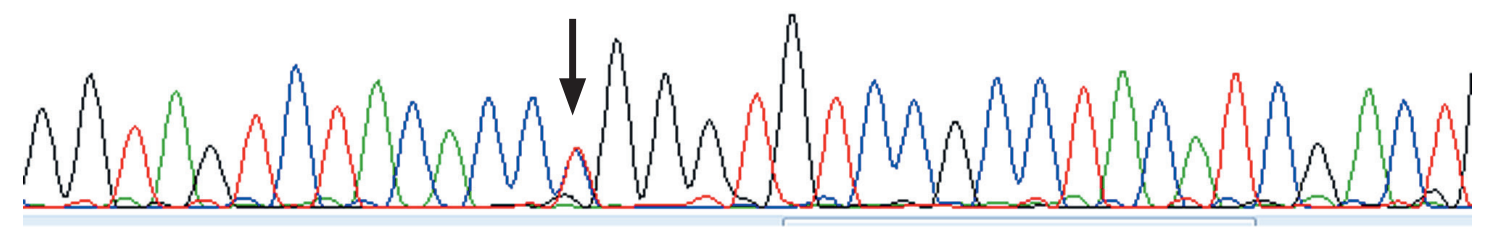

Fig. 1. Electropherogram of F strand of showing the p.Arg254Trp mutation in exon 7 of the CTRC gene. Cytosine was replaced by thymine. The affected individual is a heterozygote
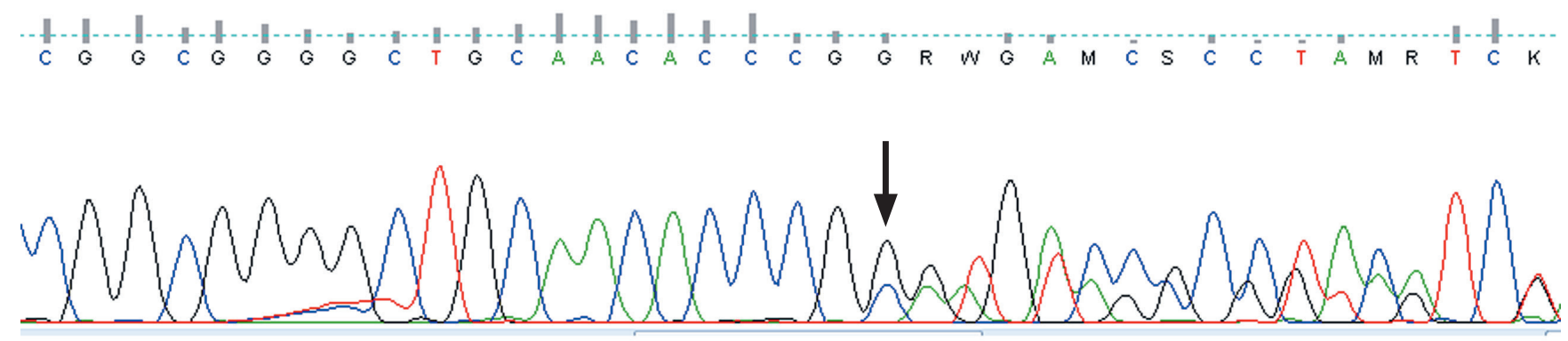

Fig. 2. Electropherogram of F strand showing the c.738_761del mutation in exon 7 of the CTRC gene. 24 bp are missing. The affected individual is a heterozygote 
Table 4. Morphological changes in the pancreas and the clinical course of ACP in the patients with and without the c.738_761del and p.Arg254Trp mutations

\begin{tabular}{|c|c|c|c|c|c|c|}
\hline \multirow{2}{*}{ Course of ACP } & \multicolumn{3}{|c|}{ c.738_761del } & \multicolumn{3}{|c|}{ p.Arg254Trp } \\
\hline & mutation (n) & no mutation (n) & $p$-value & mutation (n) & no mutation (n) & $p$-value \\
\hline Calcifications $(n=124)$ & 7 & 117 & NS & 3 & 121 & NS \\
\hline Cysts $(n=54)$ & 5 & 49 & NS & 1 & 53 & NS \\
\hline Widened Wirsung duct >3 mm $(n=36)$ & 4 & 32 & NS & 1 & 37 & NS \\
\hline Stones in Wirsung duct $(n=21)$ & 3 & 18 & NS & 2 & 19 & NS \\
\hline Operation $(n=14)$ & 5 & 9 & 0.0002 & 0 & 14 & NS \\
\hline Diabetes $(n=53)$ & 6 & 47 & 0.0484 & 2 & 51 & NS \\
\hline
\end{tabular}

ACP - alcoholic chronic pancreatitis; $n$ - number of patients; NS - no statistically significant differences.

of controls (Table 3). There were no statistically significant differences between the groups.

The c.738_761del mutation was detected in 6.98\% of women and was statistically significantly more frequent in women than in men $(0.70 \%)\left(\chi^{2}=5.130 ; p=0.0235\right)$. The p.Arg254Trp mutation was detected in $1.16 \%$ of women and in $1.41 \%$ of men, and was not statistically significantly different $\left(\chi^{2}=0.025 ; \mathrm{p}=0.8746\right)$.

The mean age at ACP onset in patients with the c.738_761del mutation was $39.87 \pm 5.56$ years and without this mutation it was $38.22 \pm 8.15$ years; with and without the p.Arg254Trp mutation, the mean age at ACP onset was $36.67 \pm 6.85$ years and $38.94 \pm 7.87$ years, respectively. The statistical analysis showed no statistically significant differences in the age at ACP onset between the patients with the c.738_761del and p.Arg254Trp mutations.

The relationship between the examined mutations and the course of ACP were analyzed with reference to morphological changes detected in the pancreas (calcifications, cysts, widening of the Wirsung duct by $>3 \mathrm{~mm}$, stones in the Wirsung duct), the occurrence of diabetes and the need for surgery. The patients with and without the c.738_761del and p.Arg254Trp mutations did not differ statistically in terms of morphological changes affecting the pancreas in the course of ACP. Fourteen patients were operated on: 2 had distal pancreatic resection, 7 had pancreaticoduodenectomy and 7 had cystojejunostomy. There were no statistical differences in the frequency of the examined mutations between patients operated on and not operated on in the course of ACP. However, the c.738_761del mutation was statistically significantly more frequent in patients with diabetes (13.21\%) in the course of ACP compared to the examined non-diabetic patients $(0 \%)(\mathrm{p}=0.0204)$; no differences were observed for the p.Arg254Trp mutation (Table 4).

\section{Discussion}

Our results revealed a frequency of $5.65 \%$ and $2.42 \%$ for the c.738_761del and p.Arg254Trp mutations in the CTRC gene, respectively, in the group of patients with ACP compared to $0 \%$ and $1.92 \%$, respectively, in the control group.
Rosendahl et al. studied the frequency of the del24 (c.738_761del) mutation in a group of German patients with ACP, and they found a $0.6 \%$ frequency of the del 24 (c.738_761del) mutation in that group compared to $0.2 \%$ with alcoholic liver disease without pancreatitis. ${ }^{13}$ According to these authors, the frequency of the R2 54W (p.Arg254Trp) mutation in other types of CP was as follows: $1.7 \%$ in idiopathic CP, $4.2 \%$ in hereditary CP and $2.1 \%$ in healthy controls. However, the del24 (c.738_761del) mutation in the German patients occurred in $1.5 \%$ of idiopathic CP cases, and in $1.2 \%$ of controls; it was not detected in the hereditary CP group. ${ }^{13}$ Chemotrypsin $\mathrm{C}$ (CTRC) variants that diminish activity or secretion are associated with chronic pancreatitis. According to Felderbauer et al., the R2 54W (p.Arg254Trp) mutation occurred in 6.5\% of the group of German patients with $\mathrm{CP}$ and hyperthyroidism, but was not detected in the group with hyperthyroidism without $\mathrm{CP}$. The authors emphasized the correlation of that mutation type and the course of CP. However, they found no other mutation types in the CTRC gene. ${ }^{14}$ A study carried out in a group of patients with primary $\mathrm{CP}$ found a frequency of $1.7 \%$ for the R2 54W (p.Arg254Trp) mutation, 0.7\% for the del24 (c.738_761del) mutation in a group of patients with idiopathic pancreatitis and $0.3 \%$ in healthy controls. ${ }^{15}$

The research results of studies on CTRC mutations in CP etiology are controversial. Some researchers believe that CTRC mutations contribute to the so-called secondary $\mathrm{CP}$ (including this of alcoholic origin), while other genetic risk factors are either absent (e.g., PRSS1 mutations) or they suggest a considerably lesser influence in comparison to primary CP (e.g., SPINK1 mutations). Therefore, those genes should be considered important contributing factors rather than causative factors. ${ }^{16,17}$ The studies in non-European populations found a higher frequency of the R2 54W (p.Arg254Trp) mutation in the group of patients with tropical pancreatitis $(2.8 \%)$ compared to healthy volunteers $(1.2 \%)$ of Indian origin. However, the del24 (c.738_761del) mutation, a considerably frequent type among German patients, was not found among Indian patients at all. Nonetheless, the interpretation of these results cannot be conclusive, because the Indian cohort was much smaller than the German group. ${ }^{13}$ The studies 
carried out in the Asian Pacific region found a significant correlation between the $C T R C$ gene mutation and tropical calcific pancreatitis. ${ }^{18}$ Considering the biochemical activity of CTRC and the functional properties of the mutation, 3 mechanisms seem to contribute to the risk of developing CP: 1. a weakened degradation of trypsinogen and/or trypsin; 2. an impaired activation of A-carbopeptidase; and 3. the induction of endoplasmic reticulum (ER) stress. Hence, the carriers of a CTRC-mutated gene are more likely to be exposed to ER stress in the exogenic region of the pancreas, which may contribute to apoptotic damage to the lobular pancreatic tissue. ${ }^{12,16,19}$

The frequency of the c.738_761del and p.Arg254Trp mutations in the CRTC gene among females and males has not been investigated so far. Our study seems to be the first in that respect. Our results found a statistically significantly higher frequency of the c.738_761del mutation in females than in males. However, no such correlation was found for the p.Arg254Trp mutation.

Most research focuses on different roles of various mutations in the etiology of pancreatitis. When a mutation is confirmed, the course of CP is rarely examined in terms of environmental and demographic factors. It seems quite interesting to learn whether a CTRC mutation quickens and affects the course of CP, e.g., by developing diabetes. Our study found that a CTRC mutation did not affect the age at ACP onset or the course of the disease.

Moreover, diabetes correlated with the c.738_761del mutation. There has been no research on CTRC gene mutations and CP course so far. However, the researchers have found that the N34S mutation in the SPINK1 gene favors ACP development and predisposes patients to developing diabetes at a younger age than patients without a mutated gene. ${ }^{5,11}$ We also found that patients with the c.738_761del mutation more often required surgery in the course of APC than patients without this mutation.

To our knowledge, this is the first study on the effect of the p.Trp55\%, p.Arg254Trp and c.738_761del mutations in the CTRC gene on the development of ACP in Polish patients. Our results are preliminary and further largepopulation studies should be conducted to confirm these findings.

\section{Conclusions}

Our results lead to following conclusions:

1. No relationship between the c.738_761del or p.Arg254Trp mutations in the CTRC gene and the development of APC or the course of APC was found in the examined group of Polish patients.

2. The mutation c.738_761del occurred statistically significantly more frequently in women than in men; however, the p.Arg254Trp mutation was not gender-dependent.
3. Neither the c.738_761del mutation nor the p.Arg254Trp mutation affected the patient's age at ACP onset or the course of the disease.

4. Contrary to p.Arg254Trp, the c.738_761del mutation in the CTRC gene correlated with diabetes development and with the need for surgery in the course of ACP.

\section{References}

1. LaRusch J, Lozano-Leon A, Stello K, et al. for the NAPS2 Consortium. The common chymotrypsinogen C (CTRC) variant G60G (C.180T) increases risk of chronic pancreatitis but not recurrent acute pancreatitis in a North American population. Clin Trans/ Gastroenterol. 2015;6(1):e68. doi: 10.1038/ctg.2014.13

2. Whitcomb DC, Yadav D, Adam S, et al. Multicenter approach to recurrent acute and chronic pancreatitis in the United States: The North American Pancreatitis Study 2 (NAPS2). Pancreatology. 2008;8:520-531.

3. Yadav D, Lowenfels AB. The epidemiology of pancreatitis and pancreatic cancer. Gastroenterology. 2013;144:1252-1261.

4. Yadaw D, Whitcomg DC. The role of alcohol and smoking in pancreatitis. Nature Rev Gastroenterol Hepatol. 2010;7:131-145.

5. Mądro A, Ciesielka M, Celiński K, et al. The genetic predisposition and its impact on the diabetes mellitus development in patients with alcoholic chronic pancreatitis. Gastroenterol Res Pract. 2015;2015:309156. http://dx.doi.org/10.1155/2015/309156

6. Global Status Report on Alcohol and Health 2011. http://www.who. int/substance_abuse/publications/global_alcohol_report/en/index. html. Accessed September 11, 2012.

7. Ravi Kanth VV, Reddy DN. Genetics of acute and chronic pancreatitis: An update. World J Gastrointest Pathophysiol. 2014;5:427-437.

8. Howell WM, Pead PJ, Shek FW, et al. Influence of cytokine and ICAM-1 gene polymorphisms on susceptibility to chronic pancreatitis. J Clin Pathol. 2005;58:595-599.

9. Shelton CA, Whitcomb DC. Genetics and treatments options for recurrent acute and chronic pancreatitis. Curr Treat Options Gastroenterol. 2014;12:359-371.

10. MasamuneA.Genetics of pancreatitis:The2014update.TohokuJExpMed. 2014;232:69-77.

11. Cichoż-Lach H, Michalak M, Lis E, et al. The N34S mutation of the SPINK1 gene and alcoholic chronic pancreatitis. Pol Arch Med Wewn. 2012;122:277-283.

12. Szmola R, Sahin-Tóth M. Chymotrypsin C (caldecrin) promotes degradation of human cationic trypsin: Identity with Rinderknecht's enzyme Y. Proc Natl Acad Sci USA. 2007;104:11227-11232.

13. Rosendahl J, Witt H, Szmola R, et al. Chymotrypsin C (CTRC) variants that diminish activity or secretion are associated with chronic pancreatitis. Nat Genet. 2008;40:78-82.

14. Felderbauer P, Karakas E, Fendrich V, Lebert R, Bartsch DK, Bulut K. Multifactorial genesis of pancreatitis in primary hyperparathyroidism: Evidence for "protective" (PRSS2) and "destructive" (CTRC) genetic factors. Exp Clin Endocrinol Diabetes. 2011;119:26-29.

15. Masson E, Chen JM, Scotet V, Le Marechal C, Ferec C. Association of rare chymotrypsinogen $C$ (CTRC) gene variations in patients with idiopathic chronic pancreatitis. Hum Genet. 2008;123:83-91.

16. Zhou J, Sahin-Tóth M. Chymotrypsin C mutations in chronic pancreatitis. J Gastroenterol Hepatol. 2011;26:1238-1246.

17. Derikx MH, Drenth JP. Genetic factors in chronic panreatitis; implications for diagnosis, management and prognosis. Best Pract Res Clin Gastroenterol. 2010;24:251-270.

18. Reddy DN, Prasad SS. Genetic basis of chronic pancreatitis in Asia Pacific region. J Gastroenterol Hepatol. 2011;26(Suppl 2):2-5.

19. Szmola R, Bence M, Carpentieri A, et al. Chymotrypsin C is a co-activator of human pancreatic procarboxypeptidases A1 and A2. J Biol Chem. 2011;286:1819-1827. 